\title{
Interactive comment on "The Vertical Variability of Black Carbon Observed in the Atmospheric Boundary Layer during DACCIWA" by Barbara Altstädter et al.
}

\section{Anonymous Referee \#1}

Received and published: 29 September 2019

This paper describes aethalometer measurements made using a UAV in west Africa. While the measurement capability is interesting, I have some fundamental concerns with the appropriateness of the aethalometer as a measurement tool and the described data treatment. Also, the paper needs to be sharpened considerably to highlight the main messages and to put them in context so that readers can easily see the value of the findings; in my opinion it will require a major revision to be publishable in ACP. Below I include, first, my major comments and then smaller issues/recommended edits.

Broader comments: 1. The microaethelometer is not a very robust tool to measure Discussion paper BC. I believe that this paper serves as the first detailed description and evaluation of 
the AE51 on ALADINA (the cited reference by Bärfuss shows a profile but says that the measurement is, as yet, unverified). There are some published studies evaluating its performance but, to my knowledge, not on airborne platforms, and often not in comparison to a verifiable BC measurement. The authors mention, at the bottom of page 4 , that the accuracy of the microaethelometer was tested in the lab. This work should be presented fully in this paper as it speaks directly to the reliability of this data.

2. Along similar lines, I am very concerned about the temperature correction. As shown it appears to be a $3 \mathrm{ug} / \mathrm{m} 3$ correction for a change of $8 \mathrm{C}$ ? Unless I'm misunderstanding that figure and associated discussion that is a huge correction factor. The correction is then bigger than most of the measurements. I can't even imagine what would cause the measurement to be temperature sensitive. Is it the light source? That would be a big change in intensity. Much more needs to be said about this. For example, is the case shown the worst-case scenario in terms of a flight with a strong temperature profile or is that typical? I think the authors should show the vertical profile of the correction factor. At a minimum, some discussion should be added describing the frequency with which large temperature corrections were applied and addressing the question of how errors in this correction factor might affect the interpretation presented here.

3. The comparison to the model is a bit odd. I can understand the value of comparing measured to modeled values but the way this analysis is written the model seems to be assumed to be correct rather than really evaluated. While I'm not sure that l'd call the AE51 "ground truth" I also doubt that the model is perfect and it seems like a stretch to me to rationalize model-measurement disagreement as artifacts. I'm not an expert in this field so I may not be aware of the literature but, have the emissions been fully validated? Has the aging scheme been compared to at least a handful of parallel observations? Have the modeled size distributions been validated? 20$25 \%$ of the BC mass in the Aitken mode seems like a lot and it seems like the model didn't represent the boundary layer very well (in that model concentrations seem to fall off while measurements are largely flat as a function of height). In the discussion

Interactive comment
Printer-friendly version

Discussion paper 
of local vs advected emissions, four reasons are given. Two are purely model based and not convincing (the model shows that the feature is regional, the model predicts mostly aged emissions). The third sounds reasonable (NOx and CO don't indicate local emissions) but those measurements should be shown and this should probably be the primary reason that advection is hypothesized. I don't really understand the 4th reason. Are there big cities or other $\mathrm{BC}$ sources between the sampling location and the coast? Otherwise I don't see how a seabreeze can bring both polluted air and clean air.

Smaller issues:

1. There are some language issues, especially towards the beginning, and I recommend English language editing. "If BC is once emitted" should be "Once BC is emitted". In the same sentence there are several unnecessary "thes", a problem which appears throughout. There are many instances where the sentence construction and grammar make it hard to understand what the authors are saying.

2. A smaller comment with regard to the data processing of the aethalometer. I don't really know what "phase shift free low pass" filtering is. The noise looks fairly random. Why not use a simple average to deal with the noisiness of the high time resolution data?

3. I'm unsure of your meaning in Line 21 with regard to hygroscopic growth. Why does hygroscopic growth enable coating?

4. There are already a lot of figures for a relatively brief paper. I don't see the value in including the power spectra graphs for the wind vectors. There you could just refer to previously published reports of that payload, especially since the present analysis doesn't seem to make use of high-resolution wind products.

5. In figure 8 , I recommend that you change the $x$-axis label in the upper right panel to say "water mixing ratio" rather than just "mixing ratio".

Printer-friendly version

Discussion paper
Interactive

comment 
6. In figure 12 you label one line as "wash out". This term is also used in the conclusions but not in the discussion. What do you mean by this? Are the low level clouds precipitating or is it just that cleaner air blew in? If it's the latter I wouldn't call that wash out, I would call it advection of cleaner air.

7. On the wind plots, how is the direction indicated by the arrows? I'm used to seeing these plotted on graphs with lat-lon as the axis but I'm not sure they make sense plotted on axes of height-time. I imagine you are just showing direction where $\mathrm{N}$ is "up" so perhaps the solution is to simply include a legend showing that? It's kind of an awkward graphic but I don't have a great solution for it.

8. There are a number of acronyms that are only used a handful of times. Notable NLLJ and LLC. Given the frequency of usage I recommend that, those just be written in full.

Interactive comment on Atmos. Chem. Phys. Discuss., https://doi.org/10.5194/acp-2019-555, 2019. 